\title{
Products and Sums of Powers of Binomial Coefficients mod $p$ and Solutions of Certain Quaternary Diophantine Systems
}

\author{
By Richard H. Hudson*
}

\begin{abstract}
In this paper we prove that certain products and sums of powers of binomial coefficients modulo $p=q f+1, q=a^{2}+b^{2}$, are determined by the parameters $x$ occurring in distinct solutions of the quaternary quadratic partition

$$
\begin{gathered}
16 p^{\alpha}=x^{2}+2 q u^{2}+2 q v^{2}+q w^{2}, \quad(x, u, v, w, p)=1, \\
x w=a v^{2}-2 b u v-a u^{2}, \quad x \equiv 4(\bmod q), \alpha \geqslant 1 .
\end{gathered}
$$
\end{abstract}

The number of distinct solutions of this partition depends heavily on the class number of the imaginary cyclic quartic field

$$
K=Q(i \sqrt{2 q+2 a \sqrt{q}}),
$$

as well as on the number of roots of unity in $K$ and on the way that $p$ splits into prime ideals in the ring of integers of the field $Q\left(e^{2 \pi i p / q}\right)$.

Let the four cosets of the subgroup $A$ of quartic residues be given by $c_{j}=2^{j} A, j=0,1,2,3$, and let

$$
s_{j}=\frac{1}{q} \sum_{t \in c_{j}} t, \quad j=0,1,2,3 .
$$

Let $s_{m}$ and $s_{n}$ denote the smallest and next smallest of the $s_{j}$ respectively. We give new, and unexpectedly simple determinations of $\prod_{k \in c_{n}} k f$ ! and $\prod_{k \in c_{n+2}} k f$ !, in terms of the parameters $x$ in the above partition of $16 p^{\alpha}$, in the complicated case that arises when the class number of $K$ is $>1$ and $s_{m} \neq s_{n}$.

1. Introduction and Summary. Throughout, $p$ will denote a prime $=q f+1$ with $q=a^{2}+b^{2} \equiv 5(\bmod 8)$ prime $, a \equiv 1(\bmod 2), b>0$. Quaternary quadratic representations of $p^{\alpha}$ or $16 p^{\alpha}, \alpha \geqslant 1$, such as

$$
\begin{gathered}
16 p^{\alpha}=x^{2}+2 q u^{2}+2 q v^{2}+q w^{2}, \quad(x, u, v, w, p)=1, \\
x w=a v^{2}-2 b u v-a u^{2}, \quad x \equiv 4(\bmod q),
\end{gathered}
$$

have been studied by, e.g., Dickson [2], Whiteman [15], Lehmer [9], Hasse [5], Giudici, Muskat, and Robinson [4], Muskat and Zee [12], and Hudson, Williams, and Buell [7]. Determination of the number of solutions (if any) of (1.1) for an arbitrary exponent $\alpha$ is a deep and complex problem as it depends on the class number of the imaginary cyclic quartic field

$$
K=Q(i \sqrt{2 q+2 a \sqrt{q}})
$$

Received December 23, 1982.

1980 Mathematics Subject Classification. Primary 10C05; Secondary 12C20, 12C25.

*Research supported by National Research Council Canada Grant \#A-7233.

(1984 American Mathematical Society $0025-5718 / 84 \$ 1.00+\$ .25$ per page 
on the number of roots of unity in $K$, and on the way that $p$ splits into prime ideals in the ring of integers of the cyclotomic field $Q\left(e^{2 \pi i p / q}\right)$.

For $q \neq 5$, the only roots of unity in $K$ are \pm 1 (see, e.g., [6, p. 4]). However, for $q=5$, there are 10 roots of unity in $K$ and (as a consequence discussed in Section 3 of [1]) the appropriate system to consider in this case is the system given first by Dickson [2], namely,

$$
\begin{gathered}
16 p^{\alpha}=x^{2}+50 u^{2}+50 v^{2}+125 w^{2}, \quad(x, u, v, w, p)=1, \\
x w=v^{2}-2 u v-u^{2}, \quad x \equiv 1(\bmod 5) .
\end{gathered}
$$

Determination of binomial coefficients of the type $\left(\begin{array}{c}r f \\ s f\end{array}\right)$ modulo $p=q f+1$, $1 \leqslant r<s \leqslant q-1$, in terms of parameters in quadratic forms has been a topic of interest since the late 1820's when Gauss [3] determined $\left(\begin{array}{c}2 f \\ f\end{array}\right)$ modulo $p=4 f+1$ in terms of the parameter $a$ in the quadratic form $p=a^{2}+b^{2}$. For a survey of known results see [8].

In [10] Emma Lehmer showed that for $p=5 f+1$ and $(x, u, v, w)$ any of the four solutions of (1.3) with $\alpha=1$ one has

$$
\left(\begin{array}{c}
2 f \\
f
\end{array}\right) \equiv-\frac{x}{2}+\frac{\left(x^{2}-125 w^{2}\right) w}{8(x w+50 u v)}(\bmod p=5 f+1)
$$

and

$$
\left(\begin{array}{c}
3 f \\
f
\end{array}\right) \equiv-\frac{x}{2}-\frac{\left(x^{2}-125 w^{2}\right) w}{8(x w+50 u v)}(\bmod p=5 f+1) .
$$

For $p=13 f+1$ and $(x, u, v, w)$ any of the four solutions of (1.1) when $\alpha=1$, Hudson and Williams [8, Theorem 16.1] proved that

$$
\left(\begin{array}{c}
4 f \\
f
\end{array}\right) \equiv-\frac{x}{2}+\frac{3\left(x^{2}-13 w^{2}\right) w}{8(x w+13 u v)}(\bmod p=13 f+1)
$$

and

$$
\left(\begin{array}{c}
7 f \\
2 f
\end{array}\right) \equiv-\frac{x}{2}-\frac{3\left(x^{2}-13 w^{2}\right) w}{8(x w+13 u v)}(\bmod p=13 f+1) .
$$

Results analogous to (1.4)-(1.7) have recently been obtained for all $q>13$; see [7, Section 6]. The starting point for these results was Matthews' [11] explicit evaluation of the quartic Gauss sum and a congruence for factorials modulo $p$ derived from the Davenport-Hasse relation in a form given by Yamamoto [16]. Using these tools and Stickelberger's theorem [14], Hudson and Williams explicitly determined $\Pi k f$ ! modulo $p=q f+1$ for all $q>5$, where $k$ runs over any of the four cosets which may be formed with respect to the subgroup of quartic residues modulo $q$, in terms of parameters in systems of the type (1.1).

We begin this paper by proving that certain products and sums of powers of products of factorials modulo $p=q f+1$ determine (and conversely are determined by) the parameters $x$ occurring in distinct solutions of (1.1) when $\alpha>1$. For example we show that

$$
\left(\begin{array}{c}
4 f \\
f
\end{array}\right)^{3}+\left(\begin{array}{c}
7 f \\
2 f
\end{array}\right)^{3} \equiv x_{3,1}(\bmod p=13 f+1)
$$




$$
\begin{aligned}
& \left(\begin{array}{c}
4 f \\
f
\end{array}\right)\left(\begin{array}{c}
7 f \\
2 f
\end{array}\right)^{2} \equiv x_{3,2}(\bmod p=13 f+1), \\
& \left(\begin{array}{c}
4 f \\
f
\end{array}\right)^{2}\left(\begin{array}{c}
7 f \\
2 f
\end{array}\right) \equiv x_{3,3}(\bmod p=13 f+1),
\end{aligned}
$$

where the $x_{k, i}, 1 \leqslant i \leqslant k$, denote from this point on the solution(s) of (1.1) when $\alpha>1$. (The subscripts will be dropped when there is no ambiguity (as when, e.g., $\alpha=1)$.)

Let the four cosets of the subgroup $A$ of quartic residues be given by $c_{j}=2^{j} A$, $j=0,1,2,3$, and let

$$
s_{j}=\frac{1}{q} \sum_{t \in c_{j}} t, \quad j=0,1,2,3 .
$$

Define $h$ to be the odd positive integer given by

$$
h=\max \left(\left|s_{0}-s_{2}\right|,\left|s_{1}-s_{3}\right|\right) .
$$

When (1.1) is solvable for $\alpha=1$, exactly four of the solutions $\left(x_{3, i}, u_{3, i}, v_{3, i}, w_{3, i}\right)$ for each $\alpha$ satisfy $x_{3, i}^{2}-q w_{3, i}^{2} \not \equiv 0(\bmod p)$ and it is convenient to let this value of $i$ be 1 . Using Stickelberger's theorem [14], Hudson and Williams [7] have shown that (1.1) is always solvable for $\alpha=h$. If $\alpha_{0}$ denotes the exponent such that (1.1) is solvable for $\alpha_{0}$ but not for $\alpha<\alpha_{0}$, we would expect to find $4 \alpha / \alpha_{0}$ solutions to (1.1) for each $\alpha$ a multiple of $\alpha_{0}$ and no solutions for $\alpha$ not a multiple of $\alpha_{0}$. This appears to be the case whenever $\left|s_{0}-s_{2}\right|=\left|s_{1}-s_{3}\right|$ and so, certainly, for all $q<101$ (as then the class number of $K$ is 1 -see [6], [13]). Moreover, this is the case for all numerical examples which may be computed by direct search techniques. A major point in this paper appears in Section 4 where we show that the unexpected does occur (and frequently). Indeed, whenever $\left|s_{0}-s_{2}\right| \neq\left|s_{1}-s_{3}\right|$ (which will always be the case when the class number is not a perfect square) and $\alpha_{0}=h$, we show that there are only $4 \alpha_{0}$ solutions to (1.1) when $\alpha=2 \alpha_{0}$. More significantly and surprisingly, the "missing" $4 \alpha_{0}$ solutions (these fail to be genuine solutions as they do not satisfy $\left.\left(x_{2,2}, u_{2,2}, v_{2,2}, w_{2,2}, p\right)=1\right)$ turn out, upon division by a certain power of $p$ to be solutions of (1.1) for $\alpha$ not a multiple of $\alpha_{0}$.

Henceforth, $s_{m}$ denotes the smallest and $s_{n}$ the next smallest of the $s_{j}$. In the closing section of this paper, Section 5 , we give new, simple, and unexpected determinations of $\prod_{k \in c_{n}} k f$ ! and $\prod_{k \in c_{n+2}} k f$ ! modulo $p$ in the most complicated case treated in [7], namely, the case that $s_{m} \neq s_{n}$.

2. Explicit Binomial Coefficient Theorems When $\alpha=2 h$ and $s_{m}=s_{n}$. Let $P_{r}$ be a prime ideal divisor of $p$ in the ring of integers of $Q\left(e^{2 \pi i p / q}\right)$. It follows from (5.33) and (5.59) of [7] that

$$
\prod_{k \in c_{m+2}} k f ! \equiv(1)^{s_{m+2}}\left(\frac{x}{2}+\frac{w}{2} \sqrt{q}\right)\left(\bmod P_{r}\right), \quad r \in c_{2-(m+2)},
$$

and

$$
\prod_{k \in c_{n+2}} k f ! \equiv(-1)^{s_{n+2}}\left(\frac{x}{2}+\frac{w}{2} \sqrt{q}\right)\left(\bmod P_{r}\right), \quad r \in c_{2-(n+2)}
$$


However, we have assumed $s_{m}=s_{n}$ in this section so we have that (having interpreted $\sqrt{q}$ as a rational expression $(\bmod p)$ and finding that $\sqrt{q}$ differs by a sign in (2.1), (2.2)- see (5.3), (5.4) of [7]),

$$
\left(\prod_{k \in c_{m+2}} k f !\right)^{2}+\left(\prod_{k \in c_{n+2}} k f !\right)^{2} \equiv \frac{x^{2}}{2}+\frac{q w^{2}}{2}(\bmod p) .
$$

Using Theorem 4.1 of [1] we now prove the following theorem.

THEOREM 2.1. There exist four solutions of (1.1) with

$$
\alpha=h=\max \left(\left|s_{0}-s_{2}\right|,\left|s_{1}-s_{3}\right|\right) \text {, }
$$

namely $\left(x_{h, 1}, u_{h, 1}, v_{h, 1}, w_{h, 1}\right),\left(x_{h, 1},-u_{h, 1},-v_{h, 1}, w_{h, 1}\right),\left(x_{h, 1}, v_{h, 1},-u_{h, 1},-w_{h, 1}\right)$, $\left(x_{h, 1},-v_{h, 1}, u_{h, 1},-w_{h, 1}\right)$ such that $p+\left(x_{h, 1}-q w_{h, 1}^{2}\right), p+\left(b x_{h, 1} w_{h, 1}+q u_{h, 1} v_{h, 1}\right)$ provided $s_{m}=s_{n}$. Let $\alpha=2 h$. Then

$$
\left(\prod_{k \in c_{m+2}} k f !\right)^{2}+\left(\prod_{k \in c_{n+2}} k f !\right)^{2} \equiv x_{2 h, 1}(\bmod p)
$$

for four solutions of (1.1) which satisfy $p+\left(x_{2 h, 1}^{2}-q w_{h, 1}^{2}\right)$ and

$$
\left(\prod_{k \in c_{m+2}} k f !\right)\left(\prod_{k \in c_{n+2}} k f !\right) \equiv x_{2 h, 2}(\bmod p)
$$

for four solutions of (1.1) which satisfy $p^{2\left(s_{n}-s_{m}\right)} \|\left(x_{2 h, 2}^{2}-q w_{2 h, 2}^{2}\right)$.

Proof. For brevity let $\left(x_{h, 1}, u_{h, 1}, v_{h, 1}, w_{h, 1}\right)=(x, u, v, w)$. Then by Theorem 4.1 of [1] we have

$$
x_{2 h, 1}=\frac{1}{4}\left(x^{2}-2 q u^{2}-2 q v^{2}-q w^{2}\right) .
$$

Clearly,

$$
x^{2}+q w^{2} \equiv-2 q u^{2}-2 q v^{2}(\bmod p)
$$

so that

$$
x_{2 h, 1} \equiv \frac{x^{2}+q w^{2}}{2}(\bmod p)
$$

and (2.4) follows immediately from (2.3). Applying the transformation $u \rightarrow v$, $v \rightarrow-u, w \rightarrow-w$, and then using (2.6) we obtain

$$
x_{2 h, 2}=\frac{x^{2}-2 q u v+2 q u v-q w^{2}}{4}=\frac{x^{2}-q w^{2}}{4} .
$$

Now (2.5) follows at once as

$$
\left(\frac{x}{2}+\frac{w}{2} \sqrt{q}\right)\left(\frac{x}{2}-\frac{w}{2} \sqrt{q}\right)=\frac{x^{2}-q w^{2}}{4} .
$$

After easy simplifications we have

$$
w_{2 h, 1}=x w \text { and } w_{2 h, 2}=-\frac{1}{2}\left(b v^{2}+2 a u v-b u^{2}\right) .
$$

Appealing to (1.1) with $\alpha=h$ (see (5.42) of [7]) we note that

$$
\left(x^{2}-q w^{2}\right)^{2}=256 p^{2 h}-64 q p^{h}\left(u^{2}+v^{2}\right)+4 q\left(b v^{2}+2 a u v-b u^{2}\right)^{2}
$$

and it follows that (see (5.40) of [7])

$$
p^{2\left(s_{n}-s_{m}\right)} \|\left(x_{2 h, 2}^{2}-q w_{2 h, 2}^{2}\right) .
$$


Moreover, we have

$$
\left(\frac{x^{2}+q w^{2}}{2}\right)^{2}-q(x w)^{2}=\frac{\left(x^{2}-q w^{2}\right)^{2}}{4}
$$

from which it follows that

$$
p+\left(x_{2 h, 1}^{2}-q w_{2 h, 1}^{2}\right)
$$

as $p^{s_{n}-s_{m}} \| b v^{2}+2 a u v-b u^{2}$ and by assumption $s_{n}=s_{m}$. Note that in [7] the signs of $a$ and $b$ are fixed to allow for a positive or negative choice of sign for $b$ in contrast to [1]. The different notations will in some cases imply a switching of roles of $u$ and $v$ in applying formulae from [7] but will not otherwise present a problem here.

Example 1. Let $q=13$ so that $s_{m}=s_{n}=1$. Then

$$
\prod_{k \in c_{2}} k f ! \equiv 4 f ! 10 f ! 12 f ! \equiv\left(\begin{array}{c}
4 f \\
f
\end{array}\right)(\bmod p)
$$

and

$$
\prod_{k \in c_{3}} k f ! \equiv 7 f ! 8 f ! 11 f ! \equiv\left(\begin{array}{c}
7 f \\
2 f
\end{array}\right)(\bmod p) .
$$

Let $p=53=4 q+1$. Then

$$
\begin{gathered}
\left(\begin{array}{c}
16 \\
4
\end{array}\right)^{2}+\left(\begin{array}{c}
28 \\
8
\end{array}\right)^{2} \equiv 18^{2}+26^{2} \equiv 6+40 \equiv 46(\bmod 53) \\
\left(\begin{array}{c}
16 \\
4
\end{array}\right)\left(\begin{array}{c}
28 \\
8
\end{array}\right) \equiv 9(\bmod 53)
\end{gathered}
$$

It is easily checked from $(2.6)$ and $(2.8)$ that $x_{2 h, 1}=-113 \equiv 46(\bmod 53)$ and $x_{2 h, 2}=9 \equiv 9(\bmod 53)$.

Example 2. Let $q=149$ so that the class number of $K$ is 9 and $s_{m}=s_{n}=17$ (see [6], [7]). A solution of (1.1) with $\alpha=h=3$ is $(-2380,2744,8824,-3392)$. Direct computation yields for $p=1193=1499 \cdot 8+1$,

$$
\prod_{k \in c_{2}} k f ! \equiv 509(1193), \quad \prod_{k \in c_{3}} k f ! \equiv 690(\bmod 1193) \text {. }
$$

From (2.6) and (2.8) we have

$$
x_{6,1}=-5931740060 \equiv 293(\bmod 1193), \quad x_{6,2}=-427169884 \equiv 486(\bmod 1193)
$$

and it is easily checked that

$$
(509)^{2}+(690)^{2} \equiv 293(\bmod 1193), \quad(509)(690) \equiv 486(\bmod 1193) .
$$

Finally,

$$
p^{2(13-12)}=1193^{2}=1423249 \mid\left(427169884^{2}-149 \cdot 521158592^{2}\right) .
$$

3. Explicit Binomial Coefficient Theorems When $\alpha=3$ and $s_{m}=s_{n}$.

THEOREM 3.1. Let $s_{m}=s_{n}$ and let $\alpha=3 h$ in (1.1). Then four solutions of (1.1) satisfy

$$
\left(\prod_{k \in c_{m+2}} k f !\right)^{3}+\left(\prod_{k \in c_{n+2}} k f !\right)^{3} \equiv x_{3 h, 1}(\bmod p),
$$

four more satisfy

$$
\left(\prod_{k \in c_{m+2}} k f !\right)\left(\prod_{k \in c_{n+2}} k f !\right)^{2} \equiv x_{3 h, 2}(\bmod p),
$$


and the remaining four solutions all have

$$
\left(\prod_{k \in c_{m+2}} k f !\right)^{2}\left(\prod_{k \in c_{n+2}} k f !\right) \equiv x_{3 h, 3}(\bmod p) .
$$

Proof. We first establish (3.1). By the binomial theorem we have

$$
\left(\frac{x}{2}+\frac{w}{2} \sqrt{q}\right)^{3}+\left(\frac{x}{2}-\frac{w}{2} \sqrt{q}\right)^{3}=\frac{x^{3}}{4}+\frac{3 q x w^{2}}{4} .
$$

Next for $(x, u, v, w)$ a solution of (1.1) when $\alpha=h$ we have from [1] that

$$
\begin{aligned}
x_{3 h, 1}= & \frac{1}{4}\left[\frac{x}{4}\left(x^{2}-2 q u^{2}-2 q v^{2}+q w^{2}\right)-\frac{2 q u}{4}(2 x u+2 b v w+2 a u w)\right. \\
& \left.\quad-\frac{2 q v}{4}(2 x v+2 b u w-2 a v w)+q w(x w)\right] \\
= & \frac{x^{3}}{16}-\frac{q x u^{2}}{8}-\frac{q x v^{2}}{16}+\frac{q x w^{2}}{16}-\frac{q x u^{2}}{4}-\frac{q b u v w}{4}-\frac{q a u^{2} w}{4} \\
& -\frac{q x v^{2}}{4}-\frac{q b u v w}{4}+\frac{q a v^{2} w}{4}+\frac{q x w^{2}}{4}
\end{aligned}
$$

as $w_{2,1}=\frac{1}{4}\left(2 x w-2 a u^{2}+2 a v^{2}-4 b u v\right)=x w$ by $(1.1)$.

However, we clearly have

$$
-\frac{3 q x u^{2}}{8}-\frac{3 q x v^{2}}{8}=\frac{3 x^{2}}{16}+\frac{3 q x w^{2}}{16}-16 p^{h}
$$

and

$$
\frac{q a v^{2} w}{4}-\frac{q b u v w}{2}-\frac{q a u^{2} w}{4}=\frac{q x w^{2}}{4} .
$$

Thus, the above equation simplifies to

$$
x_{3 h, 1}=\frac{x^{3}}{16}+\frac{5 q x w^{2}}{16}+\frac{q x w^{2}}{4}+\frac{3 x^{3}}{16}+\frac{3 q x w^{2}}{16}-16 p^{h},
$$

that is,

$$
x_{3 h, 1}=\frac{x^{3}}{4}+\frac{3 q x w^{2}}{4}-16 p^{h} .
$$

The result (3.1) is now immediate from (2.1), (2.2), (3.4) (as again we note that $\sqrt{q}$ differs by a sign in (2.1) and (2.2) when interpreted as a rational expression mod $p$ ).

Next applying the same formulae, but after first performing the transformation $u \rightarrow v, v \rightarrow-u, w \rightarrow-w$, we obtain

$$
\begin{aligned}
x_{3 h, 2}= & \frac{x\left(x^{2}-q w^{2}\right)}{16}-\frac{q x u^{2}}{8}+\frac{q b u v w}{8}+\frac{q b u^{2} w}{8}+\frac{q a u^{2} w}{8}-\frac{q a u v w}{8} \\
& -\frac{q x u v}{8}+\frac{q x u v}{8}-\frac{q b v^{2} w}{8}+\frac{q b u v w}{8}-\frac{q a u v w}{8}-\frac{q a v^{2} w}{8}-\frac{q x v^{2}}{8} \\
& +\frac{q b u^{2} w}{8}-\frac{q a u v w}{4}-\frac{q b v^{2} w}{8} .
\end{aligned}
$$

But by (1.1) we have

$$
-\frac{q x w^{2}}{8}=-\frac{q a v^{2} w}{8}+\frac{2 q b u v w}{8}+\frac{q a u^{2} w}{8} .
$$


Moreover, by (5.53) of [7] we have

$$
\frac{q b u^{2} w}{4}-\frac{q a u v w}{2}-\frac{q b v^{2} w}{4} \equiv \pm \frac{x^{2} w \sqrt{q}}{8} \pm \frac{q w^{3} \sqrt{q}}{8}(\bmod p)
$$

with the sign ambiguity resulting from the two possible sign choices for $\sqrt{q}$. Corresponding to the plus and minus choices of sign we have from (3.6) and (3.7) that

$$
x_{3 h, 2} \equiv \frac{x^{3}}{8}-\frac{q x w^{2}}{8}-\frac{x^{2} w \sqrt{q}}{8}-\frac{q w^{3} \sqrt{q}}{8}(\bmod p)
$$

and

$$
x_{3 h, 3} \equiv \frac{x^{3}}{8}-\frac{q x w^{2}}{8}+\frac{x^{2} w \sqrt{q}}{8}-\frac{q w^{3} \sqrt{q}}{8}(\bmod p) .
$$

(Verification of (3.9) using Theorem 4.1 is straightforward and left to the reader.)

The rest of the theorem now follows at once from (2.1), (2.2), upon noting that

$$
\begin{aligned}
\left(\frac{x}{2} \mp\right. & \left.\frac{w}{2} \sqrt{q}\right)\left(\frac{x}{2} \mp \frac{w}{2} \sqrt{q}\right)\left(\frac{x}{2} \pm \frac{w}{2} \sqrt{q}\right) \\
& =\frac{x^{3}}{8} \mp \frac{x^{2} w \sqrt{q}}{8}-\frac{q x w^{2}}{8} \pm \frac{q w^{2} \sqrt{q}}{8} .
\end{aligned}
$$

COROllary.

$$
x_{3 h, 2}-x_{3 h, 3}=\frac{1}{2} q w\left(b u^{2}-2 a u v-b v^{2}\right) .
$$

Proof. The expressions for $x_{3 h, 2}$ and $x_{3 h, 3}$ differ precisely by a change of sign in the expression on the left-hand side of (3.7).

Example 3. Let $q=149$ so that $a=7, b=10, s_{m}=s_{n}=17$, and a solution of (1.1) with $\alpha=h=3$ is $(-2380,2744,8824,-3392)$. Then

$$
\begin{aligned}
x_{9,1} & \equiv \frac{(-2380)^{3}}{4}+\frac{3(149)(-2380)(3392)^{2}}{4} \\
& \equiv(509)^{3}+(690)^{3} \equiv 143(\text { and } 1193),
\end{aligned}
$$

in agreement with Theorem 3.1 in view of (2.11). Moreover, appealing to (3.7), (3.8), (3.9), we have

$$
\begin{aligned}
x_{9,2} \equiv & \frac{(-2380)^{3}}{8}-\frac{149(-2380)(3392)^{2}}{8}+\frac{149(10)(2744)^{2}(-3392)}{4} \\
& -\frac{(149)(7)(2744)(8824)(-3392)}{2}-\frac{149(10)(8824)^{2}(-3392)}{4} \\
= & 27+184-228-671+151=805 \equiv(509)(509)(690)(\bmod 1193) .
\end{aligned}
$$

Finally, by (3.10) we have

$$
\begin{aligned}
x_{9,3} & \equiv 805-\frac{1}{2}(149)(-3392)(10)(2744)^{2}-(2)(7)(2744)(8824)-10(8824)^{2} \\
& \equiv 805+981(358-185-415) \equiv 810 \equiv(690)(690)(509)(\bmod 1193) .
\end{aligned}
$$

4. The Number of Solutions of (1.1) When $\alpha=2 h$ and $s_{m} \neq s_{n}$. It is exceedingly difficult to obtain numerical data giving solutions of (1.1) with $\alpha=2 h, s_{m} \neq s_{n}$. The smallest value of $q$ with $s_{m} \neq s_{n}$ is $q=101$ and the smallest prime $p=101 f+1$ is 
607. A direct search for solutions of

$$
\begin{gathered}
16(607)^{\alpha}=x^{2}+202 u^{2}+202 v^{2}+101 w^{2}, \\
x w=v^{2}-20 u v-u^{2}, \quad x \equiv 4(\bmod 101),(x, u, v, w, p)=1,
\end{gathered}
$$

is already very time consuming for $\alpha=h=3$ and appears to be hopeless for $\alpha>3$. Making use of theorems in [1] and [7], Buell and Hudson showed that

$$
(8185,-966,1971,5013)
$$

is a solution of (4.1) when $\alpha=3$ (there are no solutions when $\alpha=1$ or 2). Applying Theorem 4.1 of [1] one finds the solution

$$
\text { (407976475, 43028481, -21086784, 41031405) }
$$

for $\alpha=6$ and we note that

$$
\left(\prod_{k \in c_{n}} k f !\right)^{2} \equiv(294)^{2} \equiv 242 \equiv 407976475(\bmod 607) .
$$

However, when one applies Theorem 4.1 of [1] after applying the transformation $u \rightarrow v, v \rightarrow-u, w \rightarrow-w$ (or any of the other possible transformations) one does not obtain a solution to (1.1). Indeed in general, it follows from (2.8), (2.9) and (5.39), (5.40) of [7] that $p^{s_{n}-s_{m}} \| x_{2 h, 2}$ and $p^{s_{n}-s_{m}} \| w_{2 h, 2}$. But

$$
p^{2\left(s_{n}-s_{m}\right)}\left\|\left(x_{2 h, 2}^{2}+q w_{2 h, 2}^{2}\right) \Rightarrow p^{\left(2 s_{n}-s_{m}\right)}\right\|\left(u_{2 h, 2}^{2}+v_{2 h, 2}^{2}\right)
$$

and

$$
p^{s_{n}-s_{m}} \mid\left(b x_{2 h, 2} w_{2 h, 2}+2 q u_{2 h, 2} v_{2 h, 2}\right)
$$

by (5.40) of [7]. Together these clearly imply that

$$
p^{s_{n}-s_{m}} \mid\left(x_{2 h, 2}, u_{2 h, 2}, v_{2 h, 2}, w_{2 h, 2}\right)
$$

so that $\left(x_{2 h, 2}, u_{2 h, 2}, v_{2 h, 2}, w_{2 h, 2}, p\right) \neq 1$ if $s_{n}>s_{m}$ (that is the four-tuple obtained is not a solution of (1.1) when $\alpha=6$ in view of the restriction in (1.1) that a solution be relatively prime to $p$ ). Nonetheless, it is clear that the difficulty arises precisely because the parameters in the four-tuple have precisely $s_{n}-s_{m}$ too many $p$ 's as factors. From

$$
p^{2\left(s_{n}-s_{m}\right)} \|\left(x_{2 h, 2}^{2}+2 q u_{2 h, 2}^{2}+2 q v_{2 h, 2}^{2}+q w_{2 h, 2}^{2}\right)
$$

we see at once that

$$
\frac{1}{p^{s_{n}-s_{m}}}\left(x_{2 h, 2}, u_{2 h, 2}, v_{2 h, 2}, w_{2 h, 2}\right)
$$

is a solution of (1.1) for $\alpha=2 h-2\left(s_{n}-s_{m}\right)$. By (2.4) of [7] we have $2\left(s_{n}-s_{m}\right)<h$. Thus we have established that for $s_{n} \neq s_{m}$, the system (1.1) is not only solvable for $\alpha=h$ [7, Section 4], but also for a value of $\alpha$ that is not a multiple of $h$, namely $\alpha=2 h-2\left(s_{n}-s_{m}\right)$.

Example 4. For $q=101, p=607$, we have $s_{m}=11, s_{n}=12$ and in contrast to the case $s_{m}=s_{n}$ there appears to be only one solution to (1.1) when $\alpha=6$, namely the solution given by (4.2). However, the four-tuple

$$
\begin{aligned}
& \left(x_{2 h, 2}, u_{2 h, 2}, v_{2 h, 2}, w_{2 h, 2}\right) \\
& \quad=(-617788211,6857886,-44077305,-12854439)
\end{aligned}
$$


satisfies all the conditions of (1.1) except that each parameter is divisible by $p^{s_{n}-s_{m}}=p=607$. Consequently, the four-tuple

$$
(1017773,-11298,72615,21177)
$$

is a solution of $(1.1)$ when $\alpha=2 h-2\left(s_{n}-s_{m}\right)=6-2=4$.

5. A New Determination of Certain Products of Factorials $\bmod p=q f+1$. Extending work of Cauchy and Jacobi (who treated the quadratic case), Hudson and Williams determined in [7] the four products of factorials modulo $p=q f+1, q \equiv 5$ $(\bmod 8)>5(a$ fixed $\equiv 1(\bmod 4)$ and $b \equiv-(q-1) / 2 ! a(\bmod q))$, given by $\Pi_{k} k f !$ where $k$ runs through the four cosets which may be formed with respect to the subgroup of quartic residues modulo $q$. In particular, they showed that for $s_{m} \neq s_{n}$ (Case B in [7]) there are four solutions of (1.1) when $\alpha=h$ such that (with signs of $a, b$ fixed as above, and $x \equiv-4(\bmod q))$ one has

$$
\prod_{k \in c_{m}} k f ! \equiv \frac{(-1)^{s_{m}+1}}{x}(\bmod p),
$$

2) $\prod_{k \in c_{n}} k f ! \equiv \frac{4(-1)^{s_{n}+1}}{\left(2 x+\frac{(-1)^{(b-2(m-n)) / 4} a b w\left(x^{2}-q w^{2}\right)}{b^{2} x w+2|b| q u v}\right) / p^{s_{n}-s_{m}}}(\bmod p)$,

$$
\begin{gathered}
\prod_{k \in c_{m+2}} k f ! \equiv(-1)^{s_{m}} x(\bmod p) \\
\prod_{k \in c_{n+2}} k f ! \equiv \frac{(-1)^{s_{n}}}{4 p^{s_{n}-s_{m}}}\left(2 x+\frac{(-1)^{(b-2(m-n)) / 4} a b w\left(x^{2}-q w^{2}\right)}{b^{2} x w+2|b| q u v}\right)(\bmod p) .
\end{gathered}
$$

Obviously, the congruences (5.2) and (5.4) are rather unwieldy. As an easy consequence of the arguments in Section 2 and Section 4 of this paper we have

$$
\left(\prod_{k \in c_{n}} k f !\right)\left(\prod_{k \in c_{n+2}} k f !\right) p^{s_{n}-s_{m}} \equiv x_{2 h, 2}(\bmod p)
$$

for four solutions of (1.1) with $\alpha=2 h$ and this yields alternative determinations which are much neater as exhibited in the following theorem.

THEOREM 5.1. There are four solutions of (1.1) when $\alpha=h$, any one of which we denote by $(x, u, v, w)$, and four solutions with $\alpha=2 h-2\left(s_{n}-s_{m}\right)$ which we denote by $\left(x^{\prime}, u^{\prime}, v^{\prime}, w^{\prime}\right)$ such that for any of these 8 solutions we have

$$
\begin{gathered}
\prod_{k \in c_{m}} k f ! \equiv \frac{(-1)^{s_{m}}}{x}(\bmod p), \\
\prod_{k \in c_{n}} k f ! \equiv \frac{(-1)^{s_{m}} x}{x^{\prime}}(\bmod p), \\
\prod_{k \in c_{m+2}} k f ! \equiv(-1)^{s_{m}+1} x(\bmod p), \\
\prod_{k \in c_{n+2}} k f ! \equiv \frac{(-1)^{s_{m}+1} x^{\prime}}{x}(\bmod p) .
\end{gathered}
$$


Example 5. Let $q=101, p=607$ so that

$$
(x, u, v, w)=(8185,-966,1971,5013) \equiv(294,248,150,157)(\bmod p)
$$

and

$$
\begin{aligned}
\left(x^{\prime}, u^{\prime}, v^{\prime}, w^{\prime}\right) & =(-1017773,11298,72615,21177) \\
& \equiv(166,372,382,539)(\bmod 607) .
\end{aligned}
$$

From Example 7.1 of [7] we have

$$
(-1)^{s_{m}+1} \prod_{k \in c_{m+2}} k f ! \equiv 294(\bmod 607)
$$

and

$$
(-1)^{s_{m}+1} \prod_{k \in c_{n+2}} k f ! \equiv 302(\bmod 607) .
$$

These congruences are clearly in agreement with (5.6) and (5.8) as $(-1)^{11+1} 166 / 294$ $\equiv 302(\bmod 607)$ and $(5.6)$ follows as a consequence of (5.59) of [7].

Example 6. Let $q=157, p=1571$. Among the 12 solutions of (1.1) with $\alpha=h=3$ we have

$$
(23868,3254,8570,14948) \equiv(303,112,715,809)(\bmod 1571) .
$$

Now $\left((23868)^{2}-157(14948)^{2}\right) / 4 p^{s_{n}-s_{m}} \equiv 360(\bmod 1571)$ as $s_{0}=19, s_{1}=18, s_{2}=$ $20, s_{3}=21$ (see [7, Example 2]). Moreover,

$$
\prod_{k \in c_{m+2}} k f ! \equiv-303(\bmod 1571) \quad \text { and } \prod_{k \in c_{n+2}} k f ! \equiv 1090(\bmod 1571) .
$$

By Theorem 5.1 we should have

$$
\prod_{k \in c_{n+2}} k f ! \equiv \frac{(-1)^{19} 360}{-303} \equiv 1090(\bmod 1571),
$$

and this is easily verified.

Department of Mathematics and Statistics

University of South Carolina

Columbia, South Carolina 29208

1. Duncan A. Buell \& Richard H. Hudson, "Solutions of certain quaternary quadratic systems," Pacific J. Math., v. 114, 1984, pp. 23-45.

2. L. E. Dickson, "Cyclotomy and trinomial congruences," Trans. Amer. Math. Soc., v. 37, 1935, pp. 363-380.

3. C. F. Gauss, "Theoria residuorum biquadraticorum, Comment. I," Comment. soc. reg. sci. Gottingensis rec., v. 6, 1828, p. 27. (Werke vol. 2, p.90.)

4. Reinaldo E. Giudici, Joseph B. Muskat \& Stanley F. Robinson, "On the evaluation of Brewer's character sums," Trans. Amer. Math. Soc., v. 171, 1972, pp. 317-347.

5. Helmut HaSSE, "Der $2^{n}$-te Potenzcharakter von 2 im Körper der $2^{n}$-ten Einheitswurzeln," Rend. Circ. Mat. Palermo (2), v. 7, 1958, pp. 185-244.

6. Richard H. Hudson \& KenNeth S. Williams, A Class Number Formula for Certain Quartic Fields, Carleton Mathematical Series No. 174, Carleton University, Ottawa, 1981.

7. Richard H. Hudson, KenNeth S. Williams \& Duncan A. Buell, "Extension of a theorem of Cauchy and Jacobi," J. Number Theory (To appear.)

8. Richard H. Hudson \& KeNNETH S. Williams, "Binomial coefficients and Jacobi sums," Trans. Amer. Math. Soc., v. 281, 1984, pp. 431-505.

9. EMma LeHMer, "The quintic character of 2 and 3," Duke Math. J., v. 18, 1951, pp. 11-18.

10. EMma LeHMer, “On Euler's criterion,” J. Austral. Math. Soc., v. 1, 1959, pp. 64-70. 
11. C. R. Matrhews, "Gauss sums and elliptic functions II. The quartic sums," Invent. Math., v. 54, 1979, pp. 23-52.

12. Joseph B. Muskat \& Yun-Cheng Zee, "On the uniqueness of solutions of certain Diophantine equations," Proc. Amer. Math. Soc., v. 49, 1975, pp. 13-19.

13. BENNETT SETZER, "The determination of all imaginary, quartic, Abelian number fields with class number 1," Math. Comp., v. 35, 1980, pp. 1383-1386.

14. LOTHAR StiCKELBERGER, “Ueber eine Verallgemeinerung der Kreisteilung," Math. Ann., v.37, 1890, pp. 321-367.

15. Albert Leon Whiteman, “Theorems analogous to Jacobsthal's theorem," Duke Math. J., v. 16, 1949, pp. 619-626.

16. Когсні Үамамото, "On a conjecture of Hasse concerning multiplicative relation of Gaussian sums," J. Combin. Theory, v. 1, 1966, pp. 476-489. 